\title{
Hypothyroidism Associated with Type 2 Diabetes
}

\section{Abeer Abdul Rahman Al Saweer*}

Family and Community Medicine, Arabian Gulf University, Bahrain

\begin{abstract}
Thyroid disorders and diabetes mellitus are one of the most common duo in endocrinology. The existence of both conditions in patients seems sometimes inevitable.

Aim: To estimate the prevalence of thyroid dysfunction in type 2 diabetics in Bahrain; also to examine and compare some clinical, biometric and biochemical parameters between hypothyroid and normothyroid diabetic patients.

Methods and Material: This is part of cross sectional study that took place in 2012 and examined the prevalence of vitamin D3 in diabetics. 268 Type 2 diabetic patients' medical records were examined for history of thyroid disease. Other parameters included BMI, waist circumference, and history of hypertension, hyperlipidemia, vitamin D3 levels, glycated hemoglobin and total cholesterol.

Results: $7.83 \%(21 / 268)$ patients of this diabetic population had history of thyroid disease. Twenty patients out of $268(7.46 \%)$ had history of hypothyroidism treated with thyroid replacement therapy while $1 / 268(0.03 \%)$ had history of hyperthyroidism treated with antithyroid therapy. Total cholesterol levels, BMI and C-peptide levels were significantly higher in the hypothyroid population of diabetics compared to the normothyroid diabetics.

Conclusion: Hypothyroidism in diabetics is common problem that should be addressed and managed to avoid further complications of both endocrinological problems. This study calls for further more elaborate studies to meticulously estimate the prevalence of thyroid dysfunction in the general population and the diabetic population as well in Bahrain. Also the study reinforces the impact of thyroid dysfunction in diabetics especially in relation to cardiovascular morbidity and mortality.
\end{abstract}

Keywords: Hypothyroidism; Type 2 diabetes; Morbidity; Mortality

\section{Introduction}

Thyroid disorders and diabetes mellitus are one of the most common duo in endocrinology. The existence of both conditions in patients seems sometimes inevitable [1,2].

Diabetic patients have higher prevalence of thyroid disorders than the general population which may have an influence on diabetic management. The mismanagement of thyroid dysfunction in diabetics may cause metabolic derangement, jeopardize the glycemic control and increase cardiovascular risk. The reason for the increased prevalence of thyroid dysfunction in type 2 diabetics is under great debate. Thyroid and insulin hormones have similar impacts in many organ aspects; thus the confusion or late diagnosis of thyroid dysfunction in type 2 diabetics. The changes in thyroid hormone noticed in type 2 diabetics are decrease in stimulation of the thyroid gland by TSH, induced by central hypothyroidism, and in the peripheral conversion of $\mathrm{T}_{3}$ to $\mathrm{T}_{4}$ [1-5].

The most common type of thyroid disease is goiter, hypothyroidism and hyperthyroidism $[1,2]$.

The prevalence of thyroid disease in the region ranges from $1.7 \%$ to $47 \%$ depending on the study type and population studied. The prevalence of thyroid dysfunction in type 2 diabetics varies according to study type and origin. It may be as low as $10 \%$ or as high as $16 \%$ [6-12].

The prevalence of diabetes in Bahrain is estimated to be $14.7 \%$ according to the last Non Communicable Survey done in Bahrain. There is nevertheless scarcity in data regarding the prevalence of thyroid disease in general population in Bahrain and especially in diabetics. One study looked at thyroid goiter prevalence in Bahrain which is quoted to be $1.7 \%[13,14]$.

Though the prevalence of thyroid dysfunction is noticeable in diabetics, no international diabetes guidelines have stressed on regular monitoring of the diabetic population for the emergence of thyroid dysfunction [15].

The aim of the present study is to estimate the prevalence of thyroid dysfunction in type 2 diabetics in Bahrain. Other objectives includes examining and comparing some clinical, biometric and biochemical parameters between hypothyroid and normothyroid diabetic patients.

\section{Methodology}

This is a part of a cross sectional study that took place in May 2012 to September 2012 by the author which estimated the prevalence of vitamin $\mathrm{D}$ deficiency in type 2 diabetics in primary care center(A'ali health center) in Bahrain.

The diabetes registry in A'Ali health center in Bahrain had 1700 diabetics registered till 2011.The expected number of study participants will be at least 300 selected from the diabetes registry. The number was calculated based on the based on $99.0 \%$ as the confidence interval, total number of diabetics in the registry (1700) and an $80.0 \%$ prevalence of

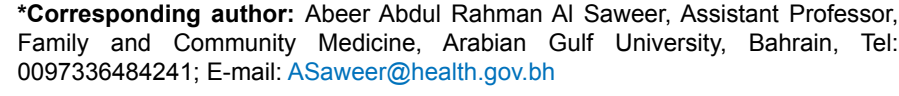

Received November 26, 2018; Accepted December 03, 2018; Published December 10, 2018

Citation: AI Saweer AAR (2018) Hypothyroidism Associated with Type 2 Diabetes. J Gen Pract 6: 369. doi: 10.4172/2329-9126.1000369

Copyright: $\odot 2018$ AI Saweer AAR. This is an open-access article distributed under the terms of the Creative Commons Attribution License, which permits unrestricted use, distribution, and reproduction in any medium, provided the original author and source are credited. 
vitamin $\mathrm{D}$ deficiency based on neighboring regional studies. The author performed analysis on the same sample and estimated the prevalence of thyroid dysfunction. Participants were randomly selected from the registry.

Parameters examined included clinical and biochemical parameters. Clinical and biometric characteristics included sex, age, BMI, waist circumference, history of treated hypertension, hyperlipidemia and IHD. Biochemical parameters included glycated haemoglobin (HbA1c), C-peptide, Vitamin D3 levels and total cholesterol levels.

Exclusion criteria were severely ill patients, pregnant women, patients with diseases other than diabetes known to be associated with vitamin $\mathrm{D}$ deficiency, such as autoimmune diseases or tuberculosis, patients on medications that interfere with vitamin $\mathrm{D}$ or calcium (steroids, antituberculous medications, Thiazides, Antacids, Phenobarbital, Phenytoin, Primidone, Valproic acid and Orlistat). Immune compromised state and acute complications of DM at the time of recruitment were also part of the exclusion criteria.

Thyroid status was retrieved from the personal interview and the medical records by the investigator. Levels were estimated as follows: Normal if TSH $\geq 0.5$ - $\leq 5 \mu \mathrm{IU} / \mathrm{ml}$, Subclinical hypothyroidism if TSH $>5-<10 \mu \mathrm{IU} / \mathrm{ml}$ ), Hypothyroidism if TSH $\geq 10$ and Hyperthyroidism if $\mathrm{TSH}<0.5 \mu \mathrm{IU} / \mathrm{ml}$.

\section{Statistical analysis}

Data was entered using SPSS version 19. Means and standard deviations were generated for age, BMI, waist circumference HbAlc, Vitamin D3 levels, C-peptide and total cholesterol. Level of glycemic control, insulin resistance, vitamin D3 level, BMI, was compared between the thyroid compromised patients and normal thyroid function patients using a t-test, as well as ANOVA test whenever appropriate. $\mathrm{P}$ value as per the original research was considered $\leq 0.5$.

\section{Ethical Approval}

The research proposal was approved by the Research Committee, Ministry of Health, Kingdom of Bahrain.

\section{Results}

Two hundred sixty-eight patients who completed the original study were included in this study. All patients completed their interview and blood tests. The average age of the population is 57.6 years $(\mathrm{SD} \pm 10.6)$. One hundred fifteen (43\%) were male; 153 (57\%) were female.

Twenty one patients $(21 / 268) 7.83 \%$ of this diabetic population had history of thyroid disease. Twenty patients out of $268(7.46 \%)$ had history of hypothyroidism treated with thyroid replacement therapy while $1 / 268(0.03 \%)$ had history of hyperthyroidism treated with antithyroid therapy.

The analysis shown below is only done for hypothothyroidism patients as there was only one case of hyperthyroidism.

Nineteen out of the 20 (95\%) patients with hypothyroidism were females and their mean age was 60.85 years $(\mathrm{SD} \pm 7.3)$.

Some clinical parameters are displayed in Table 1 which depicts the prevalence of hypertension, hyperlipidemia and ischemic heart disease (IHD) in both the hypothyroid population of diabetics and the normothyroid diabetics. It can be noticed that the prevalence of those three clinical conditions are less in the hypothyroid population compared to the normothyroid population.

\begin{tabular}{|c|c|c|}
\hline Parameter & $\begin{array}{c}\text { Hypothyroidism\% and } \\
\text { (n) }\end{array}$ & $\begin{array}{c}\text { Nonthyroiddisease } \% \\
\text { and (n) }\end{array}$ \\
\hline Hypertension & $40 \%(8 / 20)$ & $77.7 \%(192 / 247)$ \\
\hline IHD & $5 \%(1 / 20)$ & $12.5 \%(31 / 247)$ \\
\hline Hyperlipidemia & $70 \%(14 / 20)$ & $77.3 \%(191 / 247)$ \\
\hline
\end{tabular}

Table 1: Percentage of clinical parameters in thyroid compromised population.

\begin{tabular}{|c|c|c|c|}
\hline $\begin{array}{c}\text { MeanBiochemical } \\
\text { and Biometric Parameter }\end{array}$ & Hypothyroidism & $\begin{array}{l}\text { Non thyroid } \\
\text { disease }\end{array}$ & p-Value \\
\hline BMI $\left(\mathrm{kg} / \mathrm{m}^{2}\right)$ & $29.9^{*}(\mathrm{SD} \pm 6.13)$ & 31.29 & $0.46^{*}$ \\
\hline $\begin{array}{l}\text { Waist Circumference } \\
(\mathrm{cm})\end{array}$ & $102(\mathrm{SD} \pm 11.3)$ & 103.4 & 0.66 \\
\hline $\mathrm{HbA} 1 \mathrm{c}(\mathrm{mmol} / \mathrm{mmol})$ & $7.7(\mathrm{SD} \pm 2.62)$ & 7.3 & 0.6 \\
\hline C-peptide (nmol/L) & $\begin{array}{c}0.7284^{*}(S D \pm \\
0.53)\end{array}$ & $0.904(S D \pm 0.80)$ & $0.5^{*}$ \\
\hline $\begin{array}{l}\text { Total cholesterol } \\
(\mathrm{mmol} / \mathrm{L})\end{array}$ & $\begin{array}{l}4.955^{*}(S D \pm \\
1.02)\end{array}$ & 4.5 & $0.049^{*}$ \\
\hline $\begin{array}{c}\text { Vit D3level } \\
\text { (nanograms/milliliter) }\end{array}$ & $\begin{array}{c}47.85(\mathrm{SD} \pm \\
3.32)\end{array}$ & $45.33(\mathrm{SD} \pm 15.1)$ & 0.62 \\
\hline \multicolumn{4}{|c|}{ *Statistically significant } \\
\hline
\end{tabular}

Table 2: Means and SD of biometric and biochemical characteristics of the population.

Table 2 depicts relevant biometric and biochemical parameters of the hypothyroid population of diabetics and the normal thyroid function diabetics. $\mathrm{P}$ value was considered significant if it is $\leq 0.5$. BMI and C-peptide level were significantly lower in the hypothyroid population compared to the normothyroid population of the diabetics. Total cholesterol levels on the other hand were significantly higher in the hypothyroid population. Glycemic control was worse in the hypothyroid population though no significance was deducted.

\section{Discussion}

The studies looking at the prevalence of thyroid dysfunction in diabetics are scarce in this region of the world. To the author's knowledge, no other studies looked at this common endocrinological duo before in Bahrain. It is of paramount importance to examine and address this common duo because of the counteracting effects of both hormones on the function and stability of the other. This study was only able to examine the hypothyroidism and hyperthyroidism prevalence in diabetic population. Other thyroid diseases including cancer or any masses were not addressed in this diabetic population. The study was able to retrieve the prevalence through history and medical records alone. Biochemical diagnoses were not done in this population. Subclinical hypo- or hyper- thyroidism therefore was not addressed.

The prevalence of hypothyroidism in this diabetic population was $7.46 \%$ and hyperthyroidism was $0.03 \%$. In a study done in KSA which examined 200 patients with type 1 and type $2 \mathrm{DM}, 25 \%$ had subclinical hypothyroidism, 3.5\% had clinical hypothyroidism and $2.5 \%$ had clinical hyperthyroidism. In our study we did not examine the prevalence of subclinical hypothyroidism so no comparison could be done with the Saudi study $[3,11]$. The prevalence of clinical hypothyroidism was less in the Saudi study than our population. This difference may be explained by the fact that our population was older, already treated and identified for hypothyroidism. The Saudi study found the prevalence of hypothyroidism is slightly more (4\%) in type 1 compared to (3\%) in type $2 \mathrm{DM}$. Our population was solely type 2 diabetic patients; therefore no comparison can be deducted. The prevalence of clinical hyperthyroidism was much less in our study than the Saudi study. This may be explained by the fact that our population is older and has no type 1 diabetic patients $[3,11]$. 
Other studies outside the region quoted a prevalence of thyroid dysfunction in diabetics to be $14.7 \%$ with $13 \%$ suffering from clinical hypothyroidism and $12 \%$ from clinical hyperthyroidism. These figures are more than our figures and may be attributed to methodology difference and ethnicity variation [6-10,12].

Insulin resistance in the hypothyroid population of this study is lower than the normothyroid population. This is evident in the lower BMI, waist circumference and C-peptide in the hypothyroid population compared to the normothyroid population. This may be caused by the thyroid supplementation which tends to decrease weight and thus improve insulin resistance. Studies have been contradicting regarding this association, though there is tendency for increase in insulin resistance.

The glycemic control on the other hand is less well achieved in the hypothyroid population though statistically was not significant. Th is is well explained by the fact that deficiency of thyroid hormone is a known to compromise glycemic control.

Total cholesterol is significantly higher in this hypothyroid diabetic population which is more consistent with the fact that deficiency of thyroid hormone leads to dyslipidemia. This is an alarming notion since the number one killer in diabetics is cardiovascular disease and the most preventable risk factor for cardiovascular disease in diabetics is dyslipidemia.

\section{Conclusion}

This study aimed on focusing and shedding the light on the importance of thyroid dysfunction in the diabetic population in Bahrain. The interaction between both hormones is an important physiological process that is shown through cardiovascular morbidity and mortality. This study highlighted the noticeable prevalence of thyroid dysfunction in the vulnerable diabetic population. Also, it stressed on the increased cardiovascular risk in this population of hypothyroid diabetic patients.

\section{Limitation}

This study's conclusion is limited by the design of the study and number of patients. A case- control study prospective study would have validated the results and augmented its effect. If the number of patients was higher, more association could have been drawn.

\section{Recommendation}

The author recommends a more structured approach to the prevalence of thyroid conditions in diabetics with stress on subclinical hypo- and hyperthyroidism and further associates it with cardiovascular risk assessment. Also, meticulous control of thyroid conditions should be addressed to health care givers in both primary and secondary care vicinities to reduce diabetic complications and improve cardiovascular morbidity and mortality.

\section{References}

1. Palma CC, Pavesi M, Nogueira VG, Clemente EL, Vasconcellos Mde F, et al. (2013) Prevalence of thyroid dysfunction in patients with diabetes mellitus Diabetol Metab Syndr 5: 58-64.

2. Al Shahrani A, El-Metwally A, Al-Surimi K, Bin Salih S, Saleh Y, et al. (2016) The epidemiology of thyroid diseases in the Arab world: A systematic review. J Public Health Epide 8: 17-26.

3. Akbar DH, Ahmed MM, Al-Mughales J (2006) "Thyroid dysfunction and thyroid autoimmunity in Saudi type 2 diabetics," Acta Diabetol 43: 14-18.

4. Papazafiropoulou A, Sotiropoulos A, Kokoloki A, Kardara M, Stamataki Pe PS (2010) Prevalence of thyroid dysfunction among Greek type 2 diabetic patients attending an outpatient clinic. J Clin Med Res 2: 75-8.

5. Qorbani M1, Bazrafshan HR, Aghaei M, Dashti HS, Rezapour A, et al. (2013) Diabetes mellitus, thyroid dysfunctions and osteoporosis: is there an association? Send to J Diabetes Metab Disord 12: 38-43.

6. Ishay A, Chertok SI, Lavi I, Luboshitzky R (2009) Prevalence of subclinical hypothyroidism in women with type 2 diabetes. Med Sci Monit. 15: 151-155

7. Kadiyala R, Peter R, Okosieme OE (2010) Thyroid dysfunction in patients with diabetes: clinical implications and screening strategies. Int J Clin Pract 64: 1130-113.

8. Ueckermann V, Van Zyl DG (2013) The prevalence of subclinical hypothyroidism among patients with diabetes mellitus at the Kalafong Hospital Diabetes Clinic: a cross-sectional study. JEMDSA 18: 106-110.

9. Rai S, AshokKumar J, Prajna K, Shetty S, Rai T, et al. (2013) Thyroid function in type 2 diabetes mellitus and in diabetic nephropathy journal of clinical and diagnostic research. J Clin Diagn Res 7: 1583-1585.

10. Khurana A, Dhoat P, Jain G (2016) Prevalence of thyroid disorders in patients of type 2 diabetes mellitus. JIACM 17: 12-15.

11. Elmenshawi IM, Alotaibi SS, Alazmi AS, Alazmi AM, Alruwaili FR, et al. (2017) Prevalence of thyroid dysfunction in diabetic patients. Journal of Diabetes, Metabolic Disorders \& Control 4: 55-68.

12. Prasad K, Singh S (2017) Prevalence of thyroid disorders amongst patients with diabetics - a hospital based Study. Inter J Contemp Medic Res 7:1497-1499.

13. Moosa K, Abdul WA, Al-Sayyad J, Baig B (2001) National study on the prevalence of iodine deficiency disorders among schoolchildren 8-12 years of age in Bahrain. East Mediterr Health J 7: 609-616.

14. National Non-communicable Diseases Risk Factors Survey 2007.

15. American Diabetes Association. USA. http://www.diabetes.org/. Accessed on 5.3.2012. 